$1-1-2006$

\title{
Optical breathers in nonlinear anisotropic and dispersive media
}

\author{
G. T. Adamashvili \\ D. J. Kaup \\ University of Central Florida
}

Find similar works at: https://stars.library.ucf.edu/facultybib2000 University of Central Florida Libraries http://library.ucf.edu

This Article is brought to you for free and open access by the Faculty Bibliography at STARS. It has been accepted for inclusion in Faculty Bibliography 2000s by an authorized administrator of STARS. For more information, please contactSTARS@ucf.edu.

\section{Recommended Citation}

Adamashvili, G. T. and Kaup, D. J., "Optical breathers in nonlinear anisotropic and dispersive media" (2006). Faculty Bibliography 2000s. 5875.

https://stars.library.ucf.edu/facultybib2000/5875

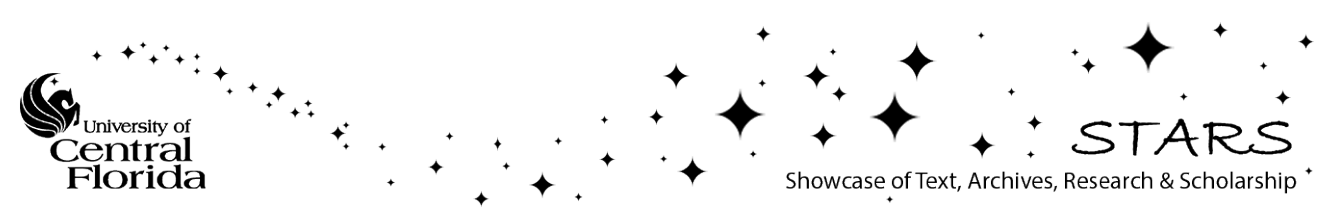




\title{
Optical breathers in nonlinear anisotropic and dispersive media
}

\author{
G. T. Adamashvili* \\ Max-Planck-Institut für Physik Komplexer System, Nöthnitzer Strasse 38, D-01187 Dresden, Germany \\ D. J. Kaup \\ Department of Mathematics and Institute for Simulation and Training, University of Central Florida, \\ Orlando, Florida 32816-1364, USA
}

(Received 10 November 2005; published 12 June 2006)

\begin{abstract}
Anisotropic crystals containing impurity atoms, when the principal optical axis of the uniaxial crystal and the vector of electrical dipole moment of the impurity atoms are perpendicular to each other and are directed along different crystallographic axes, are shown to have three different mechanisms of the formation of breathers depending on the direction of the wave propagation and on the symmetry of the medium. Explicit analytic expressions for the parameters of breathers and the effective nonlinear susceptibilities for extraordinary waves are obtained. All uniaxial crystals with quadratic susceptibilities can be divided into three different groups, according to the crystal classes. Each group is characterized by a universal structure of the breather zones. The structure of the breather zones of the trigonal, tetragonal, and hexagonal crystals with cubic susceptibilities depends neither on the crystallographic system nor on the crystal classes and coincides with the structure of the breather zones of the trigonal and hexagonal crystals with quadratic susceptibilities and crystal classes $3,3 m, \overline{6}, \overline{6} m 2$. It is shown that structure of breather zones of the different groups of crystals for the cases when the principal optical axis of the uniaxial crystal and the vector of electrical dipole moment of the impurity atoms are mutual parallel or perpendicular to each other are significantly different.
\end{abstract}

DOI: 10.1103/PhysRevE.73.066613

PACS number(s): 42.65.Tg, 42.50.Md

\section{INTRODUCTION}

An important problem in the theory of nonlinear waves in optical systems is the determination of the mechanisms of the formation of optical breathers. Depending on the nature of the nonlinearity, the nonresonance or resonance mechanism of the formation of breathers (MFB) can be realized. In the first case of nonresonant nonlinearity, which is expressed by means of the quadratic $\chi_{i j k}$ or cubic $\rho_{i j k l}$ nonlinear susceptibilities, its competition with the dispersion leads to the formation of nonresonance optical breathers [1]. The resonant optical breathers can be formed with the help of the resonance (McCall-Hahn) MFB, i.e., from a nonlinear coherent interaction of an optical pulse frequency $\omega$ which is in resonance with impurity atoms in solids when the area of the pulse $\Theta \ll 1$ and the conditions of the self-induced transparency (SIT) $\omega T \gg 1$ and $T \ll T_{1,2}$ are satisfied, where $T$ is the pulse duration, $T_{1}$ and $T_{2}$ are the longitudinal and transverse relaxation times of the impurity atoms [2-4]. In addition to these two basic mechanisms, one can have a "blended" MFB, wherein resonance and nonresonance mechanisms are acting effectively simultaneously [5-7].

The breathers [8] that we are discussing here are equivalent to the pulsating $0 \pi$ pulse from the McCall-Hahn theory of SIT and the original breather of the Sine-Gordon equation [9]. Thus they are similar to bound states of two kinklike solitons, but unlike single solitons, they can be excited with relatively low intensities of input pulses. It can also be

*Permanent address: Tbilisi State University, Chavchavadze Ave. 1, Tbilisi, Georgia 0179. shown that these breathers are related to the single soliton solution of the nonlinear Schrödinger equation (NLS) [5-7], since the NLS is the small amplitude limit of both the SineGordon equation and SIT $[4,10]$. Here, we will be interested in finding the possible breather solutions in a nonlinear solid, in the more general case when nonresonance and resonance nonlinearities and dispersion are effective.

In addition, a recent study [11] of nonresonant soliton solutions in an isotropic interacting $\chi-\rho$ system revealed many interesting aspects of their soliton solutions, among which were the fact that many solitons were unstable, and when unstable, the instability almost always led to the formation of a breather. Thus among the nonlinear objects in such systems, breathers seemed to be more stable and numerous than solitons. Consequently, one could anticipate that in such systems, breathers may generally be of more utility than single soliton solutions ( $2 \pi$ pulses).

The condition for the realization of the mechanisms of the formation of optical breathers depends on the quantities $\chi_{i j k}, \rho_{i j k l}$ and the constant of the photon-atom interaction $K=2 \pi \omega^{2} n_{0} d_{0}$ which characterizes the resonance nonlinearity, where $n_{0}$ and $d_{0}$ are the concentration and the magnitude of the vector of electrical dipole moment of the impurity atoms. Because numerical values of these quantities can vary very strongly in different media, different solids will realize different mechanisms in the formation of optical breathers.

But even more interesting for the study and comparison of different mechanisms is the investigation of these processes in one and the same crystal. Such a possibility occurs if we consider anisotropic uniaxial crystals and investigate the formation of optical breathers for optical extraordinary waves. 
It is well known that the properties of extraordinary waves depend on the direction of their propagation, and therefore different directions of propagation will incur different influences from the quantities $\chi_{i j k}, \rho_{i j k l}$, and $K$. Hence if we change the direction of the propagation of a nonlinear wave, different mechanisms in the formation of optical breathers will be realized. Consequently, in uniaxial media, there will exist certain propagation directions (and zones around them) along which one or more of the abovementioned MFB will be effective.

One of the main directions of investigation of the processes of propagation of optical breathers in anisotropic uniaxial crystals is to determine the structure of breather zones (SBZs). The investigation of the breather formation processes and specific peculiarities of the propagation of nonlinear waves in anisotropic media is also of interest, because many laser crystals are anisotropic [12], and isotropic solids can become optically anisotropic in the presence of a constant electric field or under the influence of a deformation. Different aspects of the formation and propagation of optical breathers in anisotropic crystals have been considered in detail, for one-photon processes [5,6] and two-photon excitations as well [7], under the condition when the principal optical axis $O$ of the uniaxial crystal and the vector of electrical dipole moment of the impurity atoms $\vec{d}_{0}$ coincided with each other and with a crystallographic axis. Such a situation is realized in a set of uniaxial (trigonal, tetragonal, and hexagonal) crystals, for example in materials $\mathrm{LiNbO}_{3}: \mathrm{Nd}^{3+}$, $\mathrm{CaY}_{4}\left(\mathrm{SiO}_{4}\right)_{3} \mathrm{O}: \mathrm{Nd}^{3+}, \quad \mathrm{Ba}_{2} \mathrm{ZnGe}_{2} \mathrm{O}_{7}: \mathrm{Nd}^{3+}, \quad \mathrm{SrMoO}_{4}: \mathrm{Nd}^{3+}$, and others [12].

The situation is significantly different when the principal optical axis $O$ of the uniaxial crystal and the vector of electrical dipole moment of the impurity atoms $\vec{d}_{0}$ are perpendicular to each other and are directed along different crystallographic axes. This situation is realized in a set of uniaxial (trigonal, tetragonal, and hexagonal) media, for example, in crystals $\mathrm{Al}_{2} \mathrm{O}_{3}: \mathrm{Cr}^{3+}, \mathrm{PbMoO}_{4}: \mathrm{Nd}^{3+}$, $\mathrm{CeCl}_{3}: \mathrm{Nd}^{3+}, \mathrm{CeF}_{3}: \mathrm{Nd}^{3+}, \mathrm{KY}\left(\mathrm{WO}_{4}\right)_{2}: \mathrm{Nd}^{3+}, \mathrm{LiYF}_{4}: \mathrm{Er}^{3+}$, and others.

More than this, in a set of uniaxial crystals one can have an orientation where one transition in the impurity atoms is such that the principal optical axis $O$ of the uniaxial crystal and the vector of electrical dipole moment of the impurity atoms $\vec{d}_{0}$ coincide with each other, but for other transitions in the same system, the principal optical axis $O$ of the uniaxial crystal and the vector of impurity dipole moment $\vec{d}_{0}$ can be perpendicular to each other. Such situations take place, for example, in crystals $\mathrm{CaWO}_{4}: \mathrm{Nd}^{3+}, \quad \mathrm{LiNbO}_{3}: \mathrm{Nd}^{3+}$, $\mathrm{Sr}_{5}\left(\mathrm{PO}_{4}\right)_{3} \mathrm{~F}: \mathrm{Nd}^{3+}$.

Hence investigation of the processes of the formation and propagation of optical breathers in uniaxial media when the principal optical axis $O$ of the uniaxial crystal and the vector of electrical dipole moment of the impurity atoms $\vec{d}_{0}$ are perpendicular to each other and are directed along different crystallographic axes are of interest and in need of investigation.

The main goal of this paper can be stated as follows. The investigation of the SBZ and the conditions for the

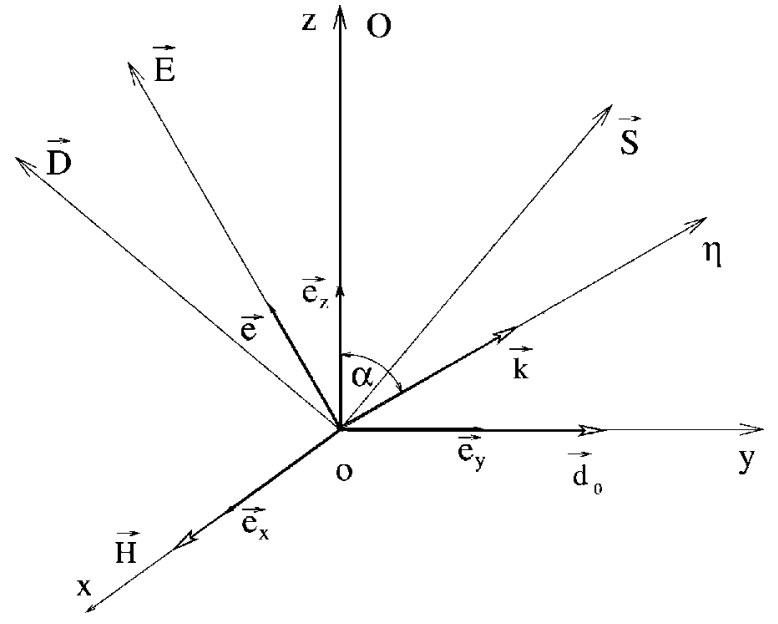

FIG. 1. The direction of the propagation of the extraordinary wave along axis $\eta$. The vectors $\vec{E}, \vec{D}, \vec{S}$, and $\vec{k}$ lie in the $y z$ plane. The principal optical axis $O$ of the uniaxial crystal points along axis $z$. The vector of electrical dipole moment of the impurity atoms $\vec{d}_{0}$ points along axis $y$. The strength of the magnetic field $\vec{H}$ points along of the direction of the axis $x$.

realization of the resonance, nonresonance, and "blended" MFB in different anisotropic uniaxial media and the determination of the explicit analytic expressions for the parameters of breathers and effective nonlinear susceptibilities for the extraordinary waves when the principal optical axis $O$ of the uniaxial crystal and the vector of electrical dipole moment of the impurity atoms $\vec{d}_{0}$ are perpendicular to each other and are directed along different crystallographic axes.

\section{BASIC EQUATIONS}

We consider the mechanisms of the formation of optical breathers in the anisotropic, (quadratic or cubic) nonlinear, and second order dispersive media containing impurity atoms in the case when an optical pulse with width $T \ll T_{1,2}$ and frequency $\omega \gg T^{-1}$ propagating in the positive direction along the $\eta$ axis. We shall consider the optically uniaxial media-trigonal, tetragonal, and hexagonal crystals with components of the permittivity tensor $\varepsilon_{x x}=\varepsilon_{y y} \neq \varepsilon_{z z}$. In these crystals, one of the principal axes of the permittivity tensor $\varepsilon_{i j}$ coincides with the axis of the symmetry of third, fourth, and sixth order, respectively. This axis is called the principal optical axis of the uniaxial crystal and we assume that this axis $O$ is pointing along $z$ axis (Fig. 1). The corresponding principal value of the tensor $\varepsilon_{i j}$ is $\varepsilon_{z z}=\varepsilon_{\|}$. Directions of the two other principal axes are pointing along $x$ and $y$ coordinate axes and coincide with two other crystallographic axes of the crystal. We designate them by $\varepsilon_{x x}=\varepsilon_{y y}=\varepsilon_{\perp}$. Without specifying the physical nature of dispersive process, we describe the dependence of permittivity tensor $\varepsilon_{i j}$ by a two-variable wave vector $\vec{k}$ and frequency $\omega$ of wave (spatially and/or temporally dispersion) [13-15]. We note that 
optics typically works with temporally dispersion, but in some special situations, spatially dispersion is also important $[16,17]$. There are two different cases: regime of normal dispersion when the group-velocity dispersion $\partial^{2} \vec{k} / \partial \omega^{2}>0$, and a regime of anomalous dispersion when this quantity is negative.

In uniaxial media, the electric displacement vector $\vec{D}$ and the vector of the strength of the electric field $\vec{E}$ of the pulse are parallel only if $\vec{k}$ points in the direction of one of the principal optical axes, but not in general. We consider situations when wave vector $\vec{k}$ always lies in the $y z$ plane. In this case there are two systems of orthogonal vector triplets $(\vec{D}, \vec{H}, \vec{k})$ and $(\vec{E}, \vec{H}, \vec{S})$ and we assume that $\vec{E}, \vec{D}, \vec{k}$, and the Poynting vector $\vec{S}=c / 4 \pi[\vec{E}, \vec{H}]$ lie in the single plane $y z$ perpendicular to the strength of the magnetic field $\vec{H}$, where $c$ is the light velocity in vacuum (Fig. 1). Then $\vec{k} \cdot \vec{r}=y k_{y}+z k_{z}$ $=k \eta$, where $\eta=z \cos \alpha+y \sin \alpha$. Thus $\eta$ is the coordinate along the direction of propagation.

The wave equation for the strength of the electrical field $\vec{E}(\eta, t)$ of the optical pulse in uniaxial media has the form

$$
\frac{\partial^{2} \vec{E}}{\partial t^{2}}-c^{2} \frac{\partial^{2} \vec{E}}{\partial \eta^{2}}=-4 \pi\left(\frac{\partial^{2} \vec{P}}{\partial t^{2}}-c^{2} \operatorname{graddiv} \vec{P}\right),
$$

where the polarization of the medium

$$
\vec{P}=\int \hat{\chi}^{(1)}\left(x_{1}, t_{1}\right) \vec{E}\left(\eta-x_{1}, t-t_{1}\right) d t_{1} d x_{1}+\vec{P}^{(2)}+\vec{P}^{(3)}+\vec{P}^{\prime} .
$$

The first-order susceptibility tensor

$$
\chi_{i j}^{(1)}=\frac{\varepsilon_{i j}-1}{4 \pi}
$$

has two independent nonzero components $\chi^{(1)}{ }_{\perp}=\chi_{x x}^{(1)}$ $=\chi^{(1)}{ }_{y y}$ and $\chi^{(1)}=\chi^{(1)}{ }_{z z}$.

The vectors $\vec{P}^{(2)}$ and $\vec{P}^{(3)}$ are nonresonant nonlinear polarizations of the second and the third orders

$$
\begin{aligned}
\vec{P}^{(2)}= & \int \hat{\chi}\left(x_{1}, x_{2}, t_{1}, t_{2}\right) \vec{E}\left(z-x_{1}, t-t_{1}\right) \vec{E}\left(z-x_{1}-x_{2}, t-t_{1}-t_{2}\right) \\
& \times d t_{1} d t_{2} d x_{1} d x_{2}, \\
\vec{P}^{(3)}= & \int \hat{\rho}\left(x_{1}, x_{2}, x_{3}, t_{1}, t_{2}, t_{3}\right) \\
& \quad \overrightarrow{\times E}\left(z-x_{1}, t-t_{1}\right) \vec{E}\left(z-x_{1}-x_{2}, t-t_{1}-t_{2}\right) \\
& \times \vec{E}\left(z-x_{1}-x_{2}-x_{3}, t-t_{1}-t_{2}-t_{3}\right) \\
& \times d x_{1} d x_{2} d x_{3} d t_{1} d t_{2} d t_{3},
\end{aligned}
$$

where $\hat{\chi}$ and $\hat{\rho}$ are the tensors of the square and cubic susceptibilities [13-15].

The quantity $\vec{P}^{\prime}$ is the resonance nonlinear polarization due to the interaction of pulse with optically active impurity atoms under condition of one-photon processes. We shall assume, as is true of a large class of laser crystals (see, for example, Ref. [12]), that the vector of electric dipole moment $\vec{d}_{0}$ of impurity atoms is perpendicular of the optical axis $O$ of uniaxial matrix. In such system the theory of self-induced transparency has been constructed in Ref. [18]. In the present work, we assume that the vector $\vec{d}_{0}$, the optical axis $O$ of matrix and the wave vector $\vec{k}$ lie in the same plane $y z$. In such a case the vector $\vec{E}$ and the vector of polarization of the impurity atoms $\vec{P}^{\prime}$ are coupling to each other through their $y$ components [18]. Consequently, we consider the nonlinear wave equation (1) for the $y$ component of $\vec{E}(\eta, t)$.

The components of the second- and third-order nonresonance nonlinear polarizations (3) have the following forms:

$$
\begin{aligned}
P_{j}^{(2)}= & \int \chi_{\mathrm{eff}, j}\left(\eta_{1}, \eta_{2}, t_{1}, t_{2}\right) E_{y}\left(\eta-\eta_{1}, t-t_{1}\right) \\
& \times E_{y}\left(\eta-\eta_{1}-\eta_{2}, t-t_{1}-t_{2}\right) d t_{1} d t_{2} d \eta_{1} d \eta_{2}, \\
P_{j}^{(3)}= & \int \rho_{\mathrm{eff}, j}\left(\eta_{1}, \eta_{2}, \eta_{3}, t_{1}, t_{2}, t_{3}\right) E_{y}\left(\eta-\eta_{1}, t-t_{1}\right) \\
& \times E_{y}\left(\eta-\eta_{1}-\eta_{2}, t-t_{1}-t_{2}\right) \\
& \times E_{y}\left(\eta-\eta_{1}-\eta_{2}-\eta_{3}, t-t_{1}-t_{2}-t_{3}\right) \\
& \times d \eta_{1} d \eta_{2} d \eta_{3} d t_{1} d t_{2} d t_{3},
\end{aligned}
$$

where

$$
\chi_{\mathrm{eff}, j}\left(\eta_{1}, \eta_{2}, t_{1}, t_{2}\right)=\chi_{j m n}\left(\eta_{1}, \eta_{2}, t_{1}, t_{2}\right) \frac{e_{E, m} e_{E, n}}{e_{E, y}^{2}}
$$

$$
\begin{aligned}
& \rho_{\mathrm{eff}, j}\left(\eta_{1}, \eta_{2}, \eta_{3}, t_{1}, t_{2}, t_{3}\right) \\
& \quad=\rho_{j m n r}\left(\eta_{1}, \eta_{2}, \eta_{3}, t_{1}, t_{2}, t_{3}\right) \frac{e_{E, m} e_{E, n} e_{E, r}}{e_{E, y}^{3}}, \quad j, n, m, r=y, z ;
\end{aligned}
$$

$\chi_{j m n}$ and $\rho_{j m n}$ are the components of tensors of the quadratic and cubic susceptibilities $[13,19,20] . e_{n, m}=\vec{e}_{n} \cdot \vec{e}_{m}, \vec{e}_{m}$ are unit vectors which are pointing along the vector $\vec{E}$ and $x, y, z$ coordinate axes; $n, m=E, x, y, z . \vec{E}=\vec{e}_{E} E, E_{z}=e_{E, z} E$. The unit vector $\vec{e}_{E}$ determines the direction of polarization of linear polarized optical wave (Fig. 1). Although for convenience in the equations, we keep both quantities $\chi_{j m n}$ and $\rho_{j m n}$, in actuality, only one of them will dominate, and depending on which, we will consider either noncentrosymmetric media or Kerr media.

Substituting the expressions (2) and (4) into the wave equation (1) and employing the condition that the field $\vec{D}$ is transverse $[\operatorname{div} \vec{D}=0$ ], we obtain the nonlinear wave equation for $E_{y}$ in the following form: 


$$
\begin{aligned}
& \frac{\partial^{2}}{\partial t^{2}} \int\left[\delta\left(\eta_{1}\right) \delta\left(t_{1}\right)+4 \pi \chi_{\perp}^{(1)}{ }_{\perp}\left(\eta_{1}, t_{1}\right)\right] E_{y}\left(\eta-\eta_{1}, t-t_{1}\right) d \eta_{1} d t_{1}-c^{2} \frac{\partial^{2}}{\partial \eta^{2}} \int\left\{\delta\left(\eta_{1}\right) \delta\left(t_{1}\right)+4 \pi \sin \alpha\left[\cos \alpha \chi_{\|}^{(1)}\left(\eta_{1}, t_{1}\right){\frac{e_{E, z}}{e_{E, y}}}{ }^{+} \sin \alpha \chi_{\perp}^{(1)}\left(\eta_{1}, t_{1}\right)\right]\right\} E_{y}\left(\eta-\eta_{1}, t-t_{1}\right) d \eta_{1} d t_{1}=-4 \pi \frac{\partial^{2}}{\partial t^{2}} \int \chi_{\mathrm{eff}, y}\left(\eta_{1}, \eta_{2}, t_{1}, t_{2}\right) E_{y}\left(\eta-\eta_{1}, t-t_{1}\right) E_{y}\left(\eta-\eta_{1}-\eta_{2}, t-t_{1}\right. \\
& \left.\quad-t_{2}\right) d t_{1} d t_{2} d \eta_{1} d \eta_{2}-4 \pi \frac{\partial^{2}}{\partial t^{2}} \int \rho_{\mathrm{eff}, y}\left(\eta_{1}, \eta_{2}, \eta_{3}, t_{1}, t_{2}, t_{3}\right) E_{y}\left(\eta-\eta_{1}, t-t_{1}\right) E_{y}\left(\eta-\eta_{1}-\eta_{2}, t-t_{1}-t_{2}\right) \times E_{y}\left(\eta-\eta_{1}-\eta_{2}-\eta_{3}, t\right. \\
& \left.\quad-t_{1}-t_{2}-t_{3}\right) d \eta_{1} d \eta_{2} d \eta_{3} d t_{1} d t_{2} d t_{3}-4 \pi \frac{\partial^{2}}{\partial t^{2}} P_{y}^{\prime}+4 \pi c^{2} \frac{\partial^{2}}{\partial \eta^{2}}\left\langle\int\left[\sin ^{2} \alpha \chi_{\mathrm{eff}, y}\left(\eta_{1}, \eta_{2}, t_{1}, t_{2}\right)+\sin \alpha \cos \alpha \chi_{\mathrm{eff}, z}\left(\eta_{1}, \eta_{2}, t_{1}, t_{2}\right)\right]\right. \\
& \left.\quad \times E_{y}\left(\eta-\eta_{1}, t-t_{1}\right) E_{y}\left(\eta-\eta_{1}-\eta_{2}, t-t_{1}-t_{2}\right) d t_{1} d t_{2} d \eta_{1} d \eta_{2}\right\rangle+4 \pi c^{2} \frac{\partial^{2}}{\partial \eta^{2}}\left\langle\int \left[\sin ^{2} \alpha \rho_{\mathrm{eff}, y}\left(\eta_{1}, \eta_{2}, \eta_{3}, t_{1}, t_{2}, t_{3}\right)\right.\right. \\
& \left.\quad+\sin \alpha \cos \alpha \rho_{\mathrm{eff}, z}\left(\eta_{1}, \eta_{2}, \eta_{3}, t_{1}, t_{2}, t_{3}\right)\right] E_{y}\left(\eta-\eta_{1}, t-t_{1}\right) E_{y}\left(\eta-\eta_{1}-\eta_{2}, t-t_{1}-t_{2}\right) E_{y}\left(\eta-\eta_{1}-\eta_{2}-\eta_{3}, t-t_{1}-t_{2}\right. \\
& \left.\left.\quad-t_{3}\right) d \eta_{1} d \eta_{2} d \eta_{3} d t_{1} d t_{2} d t_{3}\right\rangle+4 \pi c^{2} \sin ^{2} \alpha \frac{\partial^{2}}{\partial \eta^{2}}\left\langle P_{y}^{\prime}{ }_{y}\right\rangle,
\end{aligned}
$$

where

$$
\langle g\rangle=\int \varepsilon_{\|}^{-1}\left(\eta_{1}, t_{1}\right) g\left(\eta-\eta_{1}, t-t_{1}\right) d \eta_{1} d t_{1}
$$

for any function $g$.

The dependence of the quantity $P_{y}^{\prime}=n_{0} d_{0} s_{1}$ on the strength of the electrical field $E_{y}$ is governed by the optical Bloch equations which are based on the representation of the resonance impurity atoms by an ensemble of two-level atoms whose evolution is driven by the interaction with the optical waves $[21,22]$

$$
\begin{gathered}
\frac{\partial s_{1}}{\partial t}=-\omega_{0} s_{2}, \\
\frac{\partial s_{2}}{\partial t}=\omega_{0} s_{1}+\kappa_{0} E_{y} s_{3}, \\
\frac{\partial s_{3}}{\partial t}=-\kappa_{0} E_{y} s_{2},
\end{gathered}
$$

where $\kappa_{0}=2 / d_{0} \hbar, s_{i}(t)=\left\langle\hat{\sigma}_{i}(t)\right\rangle(i=1,2,3)$, where $\left\langle\hat{\sigma}_{i}\right\rangle$ is the average value of the Pauli operator $\hat{\sigma}_{i}$ and $\omega_{0}$ is the frequency of excitation of the two-level impurity atoms. We assume that $T \ll T_{1,2}$, in Eq. (6) and then neglect the relaxation effects of the impurities. Since we are assuming a small concentration of the impurity atoms, the interaction of one impurity atom with another can be ignored in the Bloch equations [21,22].

At this point, we will seek the small amplitude limit of these equations. This will be equivalent to reducing these equations to the NLS limit. To do this, we simplify Eqs. (5) and (6) by using the method of slowly varying envelopes and the dipole approximation

$$
E_{y}=\sum_{l} \hat{E}_{l} Z_{l}, \quad P_{y}^{\prime}=\frac{n_{0} d_{0}}{2} \sum_{l} Z_{l} d_{-l}\left(\delta_{l, 1}+\delta_{l,-1}\right),
$$

where $\hat{E}$ and $d_{l}=d_{l, 1}+i l d_{l, 2}$ are the slowly varying complex amplitudes of the optical wave and polarization of the impurities, and $l$ runs through the values $\pm 1, \pm 2, \ldots$. We then can write $s_{j}=\Sigma_{l} d_{l, j} Z_{l}(j=1,2)$, where $Z_{l}=e^{i l(k \eta-\omega t)}$. To guarantee the reality of the quantities $E_{y}$ and $P_{y}^{\prime}$ we set $\hat{E}_{l}=\hat{E}_{-l}^{*}$ and $d_{l}=\left(d_{-l}\right)^{*}$. As per the slowly varying envelope approximation, we assume that the envelopes $\hat{E}_{l}$ and $d_{l, i}$ vary slowly in space and time, such that $[21,22]$

$$
\begin{aligned}
& \left|\frac{\partial \hat{E}_{l}}{\partial t}\right| \ll \omega\left|\hat{E}_{l}\right|, \quad\left|\frac{\partial \hat{E}_{l}}{\partial \eta}\right| \ll k\left|\hat{E}_{l}\right|, \\
& \left|\frac{\partial d_{l, i}}{\partial t}\right| \ll \omega\left|d_{l, i}\right|, \quad\left|\frac{\partial d_{l, i}}{\partial \eta}\right| \ll k\left|d_{l, i}\right| .
\end{aligned}
$$

Substituting the expansions (7) in the system of nonlinear equations (5) and (6), we obtain for the envelopes the following nonlinear wave equation:

$$
\begin{aligned}
\sum_{l} Z_{l}\left\{\left[W_{l}(\alpha) \hat{E}_{l}-i a_{l}(\alpha) \frac{\partial \hat{E}_{l}}{\partial \eta}+i \beta_{l}(\alpha) \frac{\partial \hat{E}_{l}}{\partial t}-\mu_{l}(\alpha) \frac{\partial^{2} \hat{E}_{l}}{\partial \eta^{2}}\right.\right. \\
\left.-\gamma_{l}(\alpha) \frac{\partial^{2} \hat{E}_{l}}{\partial \eta \partial t}-\delta_{l}(\alpha) \frac{\partial^{2} \hat{E}_{l}}{\partial t^{2}}\right]-\sum_{l^{\prime}} \chi_{l, l^{\prime}}^{(2)}(\alpha) \hat{E}_{l-l^{\prime}} \hat{E}_{l^{\prime}} \\
\left.-\sum_{l^{\prime}, l^{\prime \prime}} \rho_{l, l^{\prime}, l^{\prime \prime}}^{(3)}(\alpha) \hat{E}_{l-l^{\prime}-l^{\prime \prime}} \hat{E}_{l^{\prime}} \hat{E}_{l^{\prime \prime}}-r_{l}(\alpha)\left(\delta_{l, 1}+\delta_{l,-1}\right)\right\}=0
\end{aligned}
$$

and the system of Bloch equations 


$$
\frac{\partial d_{l}}{\partial t}=i l\left(\omega_{0}-\omega\right) d_{l}+i l \kappa_{0} \hat{E}_{-l} s_{3}, \quad \frac{\partial s_{3}}{\partial t}=\frac{i l \kappa_{0}}{2}\left(d_{l} \hat{E}_{l}-d_{-l} \hat{E}_{-l}\right),
$$

where

$$
\begin{gathered}
W_{l}=l^{2}\left(c^{2} k^{2} \kappa_{l}^{(2)}-\omega^{2} \kappa_{l}^{(1)}\right), \\
a_{l}=l\left(c^{2} l k^{2} A_{l}^{(2)}+2 k c^{2} \kappa_{l}^{(2)}-l \omega^{2} A_{l}^{(1)}\right), \\
\beta_{l}=l\left(c^{2} l k^{2} B_{l}^{(2)}-2 \omega \kappa_{l}^{(1)}-l \omega^{2} B_{l}^{(1)}\right), \\
\gamma_{l}=l\left(2 \omega A_{l}^{(1)}+l \omega^{2} T_{l}^{(1)}-2 c^{2} k B_{l}^{(2)}-c^{2} l k^{2} T_{l}^{(2)}\right), \\
\delta_{l}=c^{2} l^{2} k^{2} D_{l}^{(2)}-l^{2} \omega^{2} D_{l}^{(1)}-2 l \omega B_{l}^{(1)}-\kappa_{l}^{(1)}, \\
\mu_{l}=c^{2} l^{2} k^{2} C_{l}^{(2)}+c^{2} \kappa_{l}^{(2)}+2 c^{2} l k A_{l}^{(2)}-l^{2} \omega^{2} C_{l}^{(1)}, \\
A_{l}^{(j)}=\frac{\partial \kappa_{l}^{(j)}}{\partial(l k)}, \quad B_{l}^{(j)}=\frac{\partial \kappa_{l}^{(j)}}{\partial(l \omega)}, \quad C_{l}^{(j)}=\frac{1}{2} \frac{\partial^{2} \kappa_{l}^{(j)}}{\partial(l k)^{2}}, \\
D_{l}^{(j)}=\frac{1}{2} \frac{\partial^{2} \kappa_{l}^{(j)}}{\partial(l \omega)^{2}}, \quad T_{l}^{(j)}=\frac{\partial^{2} \kappa_{l}^{(j)}}{\partial(l k) \partial(l \omega)}, \quad j=1,2 ; \\
r_{l}(\alpha)=K \psi_{l}(\alpha) d_{-l}, \quad \psi_{l}(\alpha)=1-\nu_{l} \sin ^{2} \alpha, \\
\nu_{l}=\frac{c^{2} k^{2}}{\omega^{2} \varepsilon_{\|}(l k, l \omega)}, \quad \kappa_{l}^{(1)}=\varepsilon_{\perp}(l k, l \omega), \\
\kappa_{l}^{(2)}=\int\left\{\delta\left(\eta_{1}\right) \delta\left(t_{1}\right)+4 \pi \sin ^{2} \alpha\left[\chi_{\perp}^{(1)}{ }_{\perp}\left(\eta_{1}, t_{1}\right)\right.\right. \\
\left.\left.\cot _{\|}\left(\eta_{1}, t_{1}\right) \frac{e_{E, z}}{e_{E, y}}\right]\right\} e^{i l(\omega t-k \eta)} d t d \eta .
\end{gathered}
$$

The effective susceptibilities of the second and third order in uniaxial media for $E_{y}$ have the following form:

$$
\begin{aligned}
\chi_{l, l^{\prime}}^{(2)}(\alpha)= & 4 \pi l^{2} \omega^{2}\left\{\psi _ { l } ( \alpha ) \left[\chi_{y y y}-\tan \alpha \lambda_{l}\left(\chi_{y y z}+\chi_{y z y}\right)\right.\right. \\
& \left.+\tan ^{2} \alpha \lambda_{l}^{2} \chi_{y z z}\right]-\nu_{l}\left[\sin \alpha \cos \alpha \chi_{z y y}\right. \\
& \left.\left.-\sin ^{2} \alpha \lambda_{l}\left(\chi_{z y z}+\chi_{z z y}\right)+\sin ^{2} \alpha \tan \alpha \lambda_{l}^{2} \chi_{z z z}\right]\right\},
\end{aligned}
$$

$$
\begin{aligned}
& \rho_{l, l^{\prime}, l^{\prime \prime}}^{(3)}(\alpha) \\
& \quad=4 \pi l^{2} \omega^{2}\left\{\psi _ { l } ( \alpha ) \left[\rho_{y y y y}-\tan \alpha \lambda_{l}\left(\rho_{y y y z}+\rho_{y y z y}+\rho_{y z y y}\right)\right.\right. \\
& \left.\quad+\tan ^{2} \alpha \lambda_{l}^{2}\left(\rho_{y y z z}+\rho_{y z z y}+\rho_{y z y z}\right)-\tan ^{3} \alpha \lambda_{l}^{3} \rho_{y z z z}\right] \\
& \quad-\nu_{l}\left[\sin \alpha \cos \alpha \rho_{z y y y}-\sin ^{2} \alpha \lambda_{l}\left(\rho_{z y y z}+\rho_{z y z y}+\rho_{z z y y}\right)\right. \\
& \quad+\sin ^{2} \alpha \tan \alpha \lambda_{l}^{2}\left(\rho_{z z z y}+\rho_{z z y z}+\rho_{z y z z}\right) \\
& \left.\left.\quad-\sin ^{2} \alpha \tan ^{2} \alpha \lambda_{l}^{3} \rho_{z z z z}\right]\right\},
\end{aligned}
$$

where

$$
\lambda_{l}=\frac{\varepsilon_{\perp}(l k, l \omega)}{\varepsilon_{\|}(l k, l \omega)}, \quad \chi_{i j n}=\chi_{i j n}\left(l k, l \omega, l^{\prime} k, l^{\prime} \omega\right),
$$

$$
\rho_{i j n m}=\rho_{i j n m}\left(l k, l \omega, l^{\prime} k, l^{\prime} \omega, l^{\prime \prime} k, l^{\prime \prime} \omega\right), \quad i, j, n, m=y, z .
$$

It is easy to see that all the quantities entering Eqs. (8)-(11) are dependent on the direction of wave propagation i.e., on the quantity $\alpha$. The system of Eqs. (8) and (9), for the slowly varying variables, is sufficiently general to describe the various processes of the formation and propagation of these nonlinear waves in the anisotropic, nonlinear, and dispersive media, containing small concentrations of the optical active impurity atoms, with their dipole moments pointed perpendicular to the optical axis of the host material. The expressions (10) and (11) for the effective nonlinear susceptibilities of the second and third order have general forms which are valid in any crystals in which the vector $\vec{d}$ is perpendicular of the optical axis.

For the case of trigonal, tetragonal, and hexagonal crystals where the susceptibilities of the second order have the terms $\chi_{y z z}=\chi_{z y z}=\chi_{z z y}=0$, expression (10) transforms into the form

$$
\begin{aligned}
\chi_{l, l^{\prime}}^{(2)}(\alpha)= & 4 \pi l^{2} \omega^{2}\left\{\psi_{l}(\alpha)\left[\chi_{y y y}-\tan \alpha \lambda_{l}\left(\chi_{y y z}+\chi_{y z y}\right)\right]\right. \\
& \left.-\nu_{l}\left[\sin \alpha \cos \alpha \chi_{z y y}+\sin ^{2} \alpha \tan \alpha \lambda_{l}^{2} \chi_{z z z}\right]\right\} .
\end{aligned}
$$

Similarly for third order, where the susceptibilities have the terms $\hat{\rho}_{z y z z}=\hat{\rho}_{z z z y}=\hat{\rho}_{z z y z}=\hat{\rho}_{y z z z}=0$, the effective third-order susceptibilities in Eq. (11) transform to the form

$$
\begin{aligned}
\rho_{l, l^{\prime}, l^{\prime \prime}}^{(3)}(\alpha) & \\
= & 4 l^{2} \omega^{2}\left\{\psi _ { l } ( \alpha ) \left[\rho_{y y y y}-\tan \alpha \lambda_{l}\left(\rho_{y y y z}+\rho_{y y z y}+\rho_{y z y y}\right)\right.\right. \\
& \left.+\tan ^{2} \alpha \lambda_{l}^{2}\left(\rho_{y y z z}+\rho_{y z z y}+\rho_{y z y z}\right)\right]-\nu_{l}\left[\sin \alpha \cos \alpha \rho_{z y y y}\right. \\
& \left.\left.-\sin ^{2} \alpha \lambda_{l}\left(\rho_{z y y z}+\rho_{z y z y}+\rho_{z z y y}\right)-\sin ^{2} \alpha \tan ^{2} \alpha \lambda_{l}^{3} \rho_{z z z z}\right]\right\} .
\end{aligned}
$$

\section{OPTICAL BREATHERS OF EXTRAORDINARY WAVES}

To further analyze Eqs. (8), we make use of the perturbative reduction method [23,24], in the limit that $\left|\hat{E}_{l}\right|$ is $O(\epsilon)$, with its scale-length being of order $O\left(\epsilon^{-1}\right)$. This is the typical scaling for the NLS, and as mentioned in the Introduction, would then also be the scaling for the breathers of this system.

The solution of Eqs. (8) and (9) can be carried out by two different methods depending on whether we investigate the problem of the evolution of the initial perturbation (initialvalue problem), or we consider the propagation in the medium of a pulse which is specified on the boundary of the medium (boundary-value problem). Although the corresponding equations appear different, we must note that in some sense they are identical to each other. We will investigate an initial-value problem in detail here, and we note that the boundary-value problem leads to an analogous result (see, for example, Ref. [6]).

In the case of an initial-value problem, $\hat{E}_{l}$ can be represented as 


$$
\hat{E}_{l}(\eta, t)=\sum_{j=1}^{\infty} \sum_{n=-\infty}^{+\infty} \varepsilon^{j} Y_{n} \varphi_{l, n}^{(j)}(\zeta, \tau),
$$

where $Y_{n}=e^{i n(Q \eta-\Omega t)}, \zeta=\varepsilon Q(\eta-v t), \tau=\varepsilon^{2} t, v=d \Omega / d Q$, and $\varepsilon$ is a small parameter. As such, we have an expansion in harmonics $\left(Y_{n}\right)$, and amplitudes $\left(\varepsilon^{j}\right)$, with the function $\varphi_{l, n}^{(j)}$ being the core envelope function. Consequently, consistent with the slowly varying envelope approximation, we must take

$$
\begin{gathered}
\omega \gg \Omega, \quad k \gg Q, \\
\left|\frac{\partial \varphi_{l, n}^{(\alpha)}}{\partial t}\right| \ll \Omega\left|\varphi_{l, n}^{(\alpha)}\right|, \quad\left|\frac{\partial \varphi_{l, n}^{(\alpha)}}{\partial \eta}\right| \ll Q\left|\varphi_{l, n}^{(\alpha)}\right| .
\end{gathered}
$$

In the interaction of an optical pulse with a resonantly absorbing medium, the most significant effects are usually observed at exact resonance. Therefore, for simplicity, we consider the system of Bloch equations (9) at exact resonance, i.e., with $\omega=\omega_{0}$.

To determine the explicit form of $P_{y}^{\prime}$, Eq. (2), we represent $d_{l}$ and $s_{3}$ in an amplitude perturbation series, expanding in the amplitude parameter $\varepsilon$, as

$$
d_{l}=\sum_{j=1} \varepsilon^{j} b_{l}^{(j)}, \quad s_{3}=\sum_{j=0} \varepsilon^{j} N^{(j)} .
$$

Substituting these expansions and expression (14) in Eqs. (9) we obtain

$$
\begin{aligned}
r_{l}(\alpha)= & \sum_{n} Y_{n} \frac{l}{n}\left\{R_{l}(\alpha) \sum_{j=1} \varepsilon^{j} \varphi_{l, n}^{(j)}\right. \\
& \left.-\varepsilon^{3} R_{l, 0}(\alpha) \varphi_{l, n-n^{\prime}-m}^{(1)} \varphi_{l, n^{\prime}}^{(1)} \varphi_{-l, m}^{(1)}\right\}+O\left(\varepsilon^{4}\right),
\end{aligned}
$$

where

$$
\begin{gathered}
R_{l}(\alpha)=\frac{4 \pi n_{0} d_{0}^{2} \omega^{2} \tau_{0}}{\hbar \Omega} \psi_{l}(\alpha), \\
R_{l, 0}(\alpha)=\frac{8 \pi n_{0} d_{0}^{4} \omega^{2} \tau_{0}}{(\hbar \Omega)^{3}} \psi_{l}(\alpha) .
\end{gathered}
$$

The quantity $\tau_{0}$ is simply a sign that corresponds to the initial condition in which the impurity atoms are placed. If $\tau_{0}=+1$, then the impurity atoms are initially in the ground state, i.e., as $t \rightarrow-\infty, s_{3}=-1$ (attenuating medium). The minus sign corresponds to the case when at $t \rightarrow-\infty, s_{3}=+1$, i.e., all the impurity atoms are initially in the excited state (amplifying medium). From Eqs. (15) and (16) we can see that the resonance nonlinear polarization $P_{y}^{\prime}$ or $r_{l}$ for onephoton processes (unlike two-photon processes [7]) contains not only nonlinear parts, but linear ones too.

On substituting Eqs. (14) and (15) into (8), we obtain the nonlinear wave equation

$$
\begin{aligned}
& \sum_{j, l, n} \varepsilon^{j} Z_{l} Y_{n}\left\{\left[W_{l, n}+\varepsilon J_{l, n} \partial_{\zeta}+\varepsilon^{2} H_{l, n} \partial_{\zeta \zeta}^{2}+\varepsilon^{2} h_{l, n} \partial_{\tau}+O\left(\varepsilon^{3}\right)\right] \varphi_{l, n}^{(j)}\right. \\
& \quad-\sum_{j^{\prime}, l^{\prime}, n^{\prime} j^{\prime \prime}, l^{\prime \prime}, n^{\prime \prime}} \varepsilon^{j^{\prime}}\left[\chi_{2, l, l^{\prime}} \varphi_{l-l^{\prime}, n-n^{\prime}}^{(j)} \varphi_{l^{\prime}, n^{\prime}}^{\left(j^{\prime}\right)}\right. \\
& \left.\left.+\varepsilon^{j^{\prime \prime}} \rho_{3, l, l^{\prime}, l^{\prime \prime}} \varphi_{l-l^{\prime}-l^{\prime \prime}, n-n^{\prime}-n^{\prime \prime}}^{(j)} \varphi_{l^{\prime}, n^{\prime}}^{\left(j^{\prime}\right)} \varphi_{l^{\prime \prime}, n^{\prime \prime}}^{\left(j^{\prime \prime}\right)}\right]\right\} \\
& \quad-\sum_{l, n} Z_{l} Y_{n} \frac{l}{n}\left[R_{l} \sum_{j} \varepsilon^{j} \varphi_{l, n}^{(j)}-\varepsilon^{3} R_{l, 0} \varphi_{l, n-n^{\prime}-m}^{(1)} \varphi_{l, n^{\prime}}^{(1)} \varphi_{-l, m}^{(1)}\right. \\
& \left.+O\left(\varepsilon^{4}\right)\right]=0,
\end{aligned}
$$

where

$$
\begin{gathered}
W_{l, n}=W_{l}+a_{l} n Q+\beta_{l} n \Omega-\gamma_{l} n^{2} Q \Omega+\mu_{l} n^{2} Q^{2}+\delta_{l} n^{2} \Omega^{2} \\
J_{l, n}=-i Q\left[a_{l}+\beta_{l} v_{g}+2 n Q \mu_{l}+2 n \delta_{l} \Omega v_{g}-\gamma_{l} n\left(\Omega+Q v_{g}\right)\right] \\
H_{l, n}=Q^{2}\left(\gamma_{l} v_{g}-\mu_{l}-\delta_{l} v_{g}^{2}\right) \\
h_{l, n}=i\left(\beta_{l}+2 n \Omega \delta_{l}-n Q \gamma_{l}\right)
\end{gathered}
$$

are dependent upon $\alpha$.

To determine the values of $\varphi_{l, n}^{(j)}$ we equate to zero the various terms corresponding to same powers of $\varepsilon$. As a result, we obtain a chain of equations. Starting with first order in $\varepsilon$, we have

$$
w_{l, n} \varphi_{l, n}^{(1)}=0,
$$

where

$$
w_{l, n}(\alpha)=W_{l, n}(\alpha)+\frac{l}{n} R_{l}(\alpha) .
$$

In dispersive media, we have that $W_{0}=W_{ \pm 1}=0$ while $W_{|l|>1} \neq 0$. Thus $W_{l= \pm 1}=0$ provides the dispersion law for extraordinary waves, which is

$$
\frac{c^{2} k^{2}}{\omega^{2}}=\frac{\varepsilon_{\|} \varepsilon_{\perp}}{\varepsilon_{\perp}+\cos ^{2} \alpha\left(\varepsilon_{\|}-\varepsilon_{\perp}\right)} .
$$

In what follows, we shall also be interested in localized breathers which vanish as $t \rightarrow \pm \infty$. Consequently, according to Eq. (19), only the following components of $\varphi_{l, n}^{(1)}$ can differ from zero: $\varphi_{ \pm 1, \pm 1}^{(1)}$ or $\varphi_{ \pm 1, \mp 1}^{(1)}$. The relation between $\Omega$ and $Q$, for fixed values of $l$ and $n= \pm 1$, is determined from Eq. (19) and has the form

$$
a_{l} n Q+\beta_{l} n \Omega-\gamma_{l} n^{2} Q \Omega+\mu_{l} n^{2} Q^{2}+\delta_{l} n^{2} \Omega^{2}+\frac{l}{n} R_{l}=0 .
$$

All coefficients of this equation are functions of $\alpha$ and hence the relation between $\Omega$ and $Q$ will also depend on the angle $\alpha$ too.

We have to consider the situation when $\varphi_{ \pm 1, \pm 1}^{(1)}=0$ and $\varphi_{ \pm 1, \mp 1}^{(1)} \neq 0$ as a separate case from the situation when $\varphi_{ \pm 1, \pm 1}^{(1)} \neq 0$ and $\varphi_{ \pm 1, \mp 1}^{(1)}=0$. First we consider the situation 
when $\varphi_{+1, \mp 1}^{(1)}=0, \varphi_{ \pm 1, \pm 1}^{(1)} \neq 0$ in detail. Then the relation between $\Omega$ and $Q$ is determined from Eq. (22) at $l=n= \pm 1$.

Analogously results will occur when $\varphi_{ \pm 1, \mp 1}^{(1)} \neq 0$ and $\varphi_{ \pm 1, \pm 1}^{(1)}=0$ and $l=-n= \pm 1$ [6]. In this case the terms proportional to the $R_{l}$ and $R_{l, 0}$ in Eqs. (17), (20), and (22) will change sign in comparison with the previous case because the ratio $l / n$ is now negative.

Substituting Eqs. (21) and (22) into Eq. (18), we can easily see that $J_{ \pm 1, \pm 1}=0$. To second order in $\varepsilon$, from Eq. (17) we obtain the relation between $\varphi_{ \pm 2, \pm 2}^{(2)}$ and $\varphi_{ \pm 1, \pm 1}^{(1)}$

$$
\varphi_{ \pm 2, \pm 2}^{(2)}=\frac{\chi_{ \pm 2, \pm 1}^{(2)}}{w_{ \pm 2, \pm 2}}\left(\varphi_{ \pm 1, \pm 1}^{(1)}\right)^{2} .
$$

Substituting Eqs. (22) and (23) into Eq. (17), to third order in $\varepsilon$, we finally obtain the NLS for $\Psi_{l}=\varepsilon \sqrt{q_{l}} \varphi_{l, l}^{(1)}$ (for $l= \pm 1$ ):

$$
i l \frac{\partial \Psi_{l}}{\partial t}+\frac{\partial^{2} \Psi_{l}}{\partial y_{l}^{2}}+\left|\Psi_{l}\right|^{2} \Psi_{l}=0,
$$

where

$$
\begin{gathered}
y_{l}=\frac{\eta-v_{g} t}{\sqrt{p_{l}}}, \quad p_{l}=\frac{i l H_{l, l}}{h_{l, l} Q^{2}}=-\frac{1}{2} \frac{\partial^{2} \Omega}{\partial Q^{2}}, \\
q_{l}=\frac{m_{l}+R_{l, 0}}{2 \Omega \delta_{l}+l \beta_{l}-Q \gamma_{l}} .
\end{gathered}
$$

In the above,

$$
m_{l}=M_{l}=\frac{\left(\chi_{l,-l}^{(2)}+\chi_{l, 2 l}^{(2)}\right) \chi_{2 l, l}^{(2)}}{w_{2 l, 2 l}}
$$

is the nonresonance nonlinear term for quadratic nonlinearity crystals, while

$$
m_{l}=L_{l}=\rho_{l, l,-l}^{(3)}+\rho_{l,-l, l}^{(3)}
$$

is the same for crystals with cubic nonlinearity.

The NLS (24) under the condition $p_{l} q_{l}>0$ has the soliton solution

$$
\Psi_{l}=2 i l \eta_{0} \frac{e^{-i l \varphi_{1, l}}}{\cosh 2 \eta_{0} \varphi_{2, l}},
$$

where $\Psi$ is the envelope of the soliton and

$$
\begin{gathered}
\varphi_{1, l}=\frac{2 \xi_{0} \eta}{\sqrt{p_{l}}}+2\left[2\left(\xi_{0}^{2}-\eta_{0}^{2}\right)-\frac{\xi_{0} v_{g}}{\sqrt{p_{l}}}\right] t-\varphi_{0}, \\
\varphi_{2, l}=\frac{\eta}{\sqrt{p_{l}}}+\left(4 \xi_{0}-\frac{v_{g}}{\sqrt{p_{l}}}\right) t-y_{0} .
\end{gathered}
$$

The quantities $\xi_{0}, \eta_{0}, \varphi_{0}$, and $y_{0}$ are the scattering data, which arise when the NLS is solved by the inverse scattering transform (IST) [24-26]. Substituting the soliton solution for $\Psi$, Eq. (25), into Eq. (14), at $l=n= \pm 1$ and $q_{l} \neq 0$, we obtain for the envelope for $\hat{E}_{l}$, for this breather solution

$$
\hat{E}_{l}=\frac{2 i l \eta_{0}}{\sqrt{q_{l}}} \frac{e^{-i l\left(\varphi_{1, l}+\Omega t-Q \eta\right)}}{\cosh 2 \eta_{0} \varphi_{2, l}} .
$$

From the IST, we know that arbitrary initial data for $\Psi(t=0, \eta)$ can be resolved into its component parts of solitons and radiation (continuous spectra). Given the initial data for $\hat{E}(t=0, \eta)$, by the above, one can also obtain the initial data for $\Psi(t=0, \eta)$, and thereby obtain the soliton and the radiation spectra. A sufficient condition for a soliton to be present is simply for the area of $\Psi(t=0, \eta)$ to be greater than $\pi$, provided that $\Psi(t=0, \eta)$ has only one maximum or minimum.

The appearance in expression (27) of the factor $e^{i l(Q \eta-\Omega t)}$ indicates the presence of periodic beats (slow in comparison with the corresponding optical quantities, with characteristic parameters $\Omega$ and $Q$ ), as a result of which the soliton solution (25) for $\Psi_{l}$ is transformed into the solution (27) for complex envelope $\hat{E}_{l}$. This is a closed-form time (and/or space) solution of the nonlinear wave equation (17). In the frame of the NLS, it is called simply a soliton, but in the frame of SIT or the Sine-Gordon equation, it would be called a breather (pulsing soliton), with the NLS soliton being the envelope. In either frame, to the order that these equations have been expanded, the object will propagate through the medium without any loss of energy and will be stable against infinitesimal perturbations of the initial data $[10,25]$. Equation (27) is a breather in this sense, which is simply an NLS soliton as the envelope, with the phase modulation being given by $\left(\varphi_{1, l} \neq 0\right)$. From Eqs. (10)-(13), (18), (21), (22), (26), and (27), we can see that all parameters of the breather will depend on the direction of the wave propagation.

The stability of the breather solution as it propagates in a solid is a question that has not been addressed in this paper. Such would require one to carry out the expansion done herein to one additional order. Doing so would give one a collection of various terms involving damping, anisotropy, diffraction, which would act on the breather as it propagates. Once such terms would be obtained, then there are standard methods for determining their effects on a breather or soliton $[27,28]$.

\section{SBZ WHEN $\vec{d}_{0}$ POINT ALONG AXIS $y$}

In the present paper we have shown that in the propagation of optical pulse through anisotropic uniaxial crystals with second order (temporally and/or spatially) dispersion $\left(\partial^{2} \vec{k} / \partial \omega^{2} \neq 0\right)$ and (second or third order) nonlinearities containing small concentration of the optical resonance impurity atoms, optical breathers can arise. The explicit form of the breather is given by Eq. (27). The dispersion equation and the relation between $\Omega$ and $Q$ are given by Eqs. (21) and (22). This statement is valid if the condition $q_{l} p_{l}>0$ is satisfied.

The quantity $q_{l}$ contains terms coming from both the resonance $R_{l, 0}$ and nonresonance $m_{l}$ nonlinear terms and depending on the values of these quantities, different mechanisms of the formation of optical breathers can take place.

(a) $m_{l}=R_{l, 0}, q_{l} \neq 0$, and $m_{l} R_{l, 0}>0$. This is the condition of the realization of the "blended" MFB when both the nonresonance and resonance nonlinearities are simultaneously effective and act together with the dispersion in the process of the 


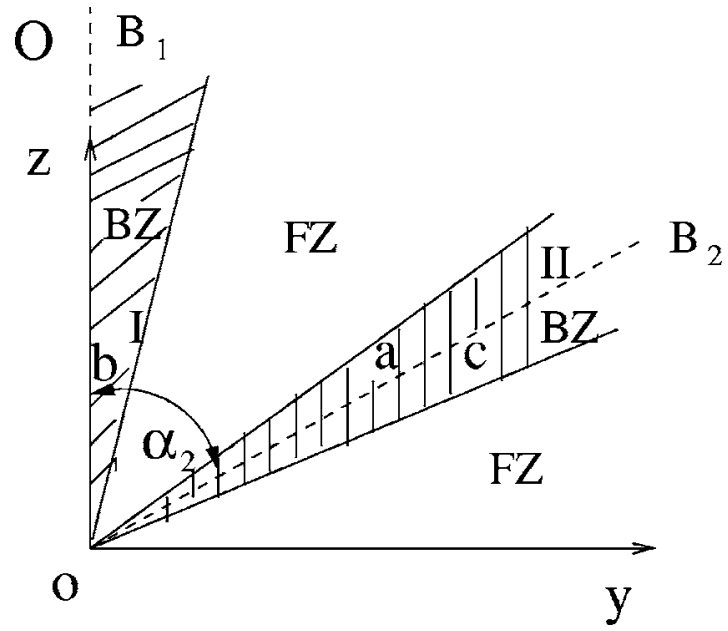

FIG. 2. There are two different characteristic directions $B_{1}$ and $B_{2}$ shown as dashed lines. The direction $B_{1}$ coincides with $z$ axis. The breather zones (BZ) correspond to the hatched regions. In the forbidden zone (FZ) regions, the MFB is suppressed. The symbols $a, b$, and $c$ indicate which if the three different mechanisms, (a), (b), and (c), are operative in that region.

formation of resonance optical breathers Eq. (see also Ref. [6]).

(b) $m_{l} \ll R_{l, 0}$. The interaction of the pulse with optical impurities dominates and nonresonance interactions can be ignored. This situation corresponds to the SIT in dispersive medium and resonant optical breathers [4].

(c) $m_{l} \gg R_{l, 0}$. The interaction of the pulse with optical impurities is negligible and at most, it causes a renormalization of their parameters [1]. In particular, in Eq. (22), which determines the connection between $\Omega$ and $Q$, we should substitute $R_{l, 0}=0$ (see Ref. [1]).

From the expressions (10)-(13) and (16), it is clear that the quantities $m_{l}$ and $R_{l, 0}$ depend not only differently on the direction of wave propagation, but also on the symmetry of the medium. Hence the mechanisms of the formation of the optical breathers of extraordinary waves, which are determined by means of the quantities $m_{l}$ and $R_{l, 0}$ will depend both on the direction of propagation of the pulses and on the symmetry of the medium. Thus we expect that several directions for wave propagation can exist in uniaxial crystals, in which different mechanisms [(a), (b), and (c)] will contribute differently to the formation of the optical breathers. We shall call these directions "characteristic directions for breathers." In order to find these directions, it will be necessary to analyze the symmetry of the media.

Let us next consider (noncentrosymmetric) uniaxial crystals with quadratic nonlinearity in the angle interval $[0, \pi / 2]$. First, for $\alpha$ near zero, the quantity $\psi_{l}(0) \approx 1$, and hence the quantity $R_{l, 0}(0)$ takes its maximum value. Under this condition the phenomenon of the SIT [MFB (b)] is most effective and resonant optical breathers of small area can be formed, wherein the characteristic direction coincides with $z$ axis. This direction is shown in Fig. 2 as the direction $B_{1}$.

An important point is that from Eq. (12) we see that depending on the symmetry of the crystals, the quantity $\chi_{l, l^{\prime}}^{(2)}(\alpha \rightarrow 0)$ can take on various values. Also, in this limit, it will mainly be determined by $\chi_{y y y}$.
For the trigonal, tetragonal, and hexagonal crystal systems with crystal classes (CC) $32,422, \overline{42} \mathrm{~m}$, and 622 , all considered components of the quadratic susceptibility tensor, and hence the quantity $\chi_{l, l^{\prime}}^{(2)}$, vanishes [13]. Thus, in these crystals, only the SIT $[\mathrm{MFB}(\mathrm{b})]$ can take place. For the $\mathrm{CC} 4, \overline{4}$, $4 \mathrm{~mm}$, in the tetragonal crystals and 6,6mm in the hexagonal crystals, for which the component $\chi_{y y y}=0$ and the quantity $\chi_{l, l^{\prime}}^{(2)}(\alpha \rightarrow 0)=0$, only MFB (b) will be effective. But the situation will be different for the CC $3,3 m$ and $\overline{6}, \overline{6} m 2$ of the trigonal and hexagonal, for in these crystals, the quantity $\chi_{l, l^{\prime}}^{(2)}(\alpha \rightarrow 0) \neq 0$ and depending on the ratio $m_{l} / R_{l, 0}$, either (b) or (a) MFB will be effective.

Second, for $\alpha$ near $\pi / 2$, the quantity $E_{y}$ tends to vanish, and hence for this direction, the expressions (10) and (11) [or Eqs. (12) and (13)] are not determined. (Note that we could have considered the wave equation (1) for the $z$ component of the vector $\vec{E}$, instead of the $y$ component, and then instead of Eqs. (12) and (13), we would have had analogous expressions for $E_{z}$. This is done in the next section [see Eqs. (30) and (31) and also Refs. [6,7]].) Thus for this direction, $\psi_{l}(\pi / 2)=R_{0, l}(\pi / 2)=0$. Therefore for $\alpha$ close to $\pi / 2$, the quantity $E_{y}$ is very small and for these directions, no breathers can be expected to exist. With decreasing $\alpha$, the quantities $E_{y}, \psi_{l}(\alpha)$, and $R_{0, l}(\alpha)$ begin also to increase. Since the quantity $R_{l, 0}$ remains small, the influence of the impurities on the wave processes also remains small, and hence they do not contribute to the formation of breathers. Thus in this region we still do not find that breathers exist, not only for the trigonal, tetragonal, and hexagonal crystal systems with crystal classes (CC) 32, 422, $\overline{42} \mathrm{~m}$, and 622 , for which all considered components of the quantity $\chi_{l, l^{\prime}}^{(2)}$ equal zero and for crystals with CC $\overline{4}, \overline{6}$, and $\overline{6} m 2$ for which $\chi_{z z z}=0$, but also for any other crystals, even those in which $\chi_{z z z} \neq 0$, because when $M_{l} w_{2 l, 2 l}<0$ then $p q<0$. So with $\alpha$ is close to $\pi / 2$, we do not expect to find any breathers.

The third important direction is $\alpha \approx \alpha_{2}$, where $\alpha_{2}$ is defined to be that value of $\alpha$ such that $\chi_{2}\left(\alpha_{2}\right)$ has its maximum positive value. Now depending on the ratio $m_{l} / R_{l, 0}$, either (a) or (c) MFB will be realized. Such a situation can be realized in all trigonal, tetragonal and hexagonal crystals, except crystals with CC $32,422, \overline{42} \mathrm{~m}$, and 622 . This direction is shown in Fig. 2 as $B_{2}$.

Now, in crystals with quadratic nonlinearity, in the general case, we already have two characteristic directions $B_{1}$ and $B_{2}$ (see Fig. 2), except for crystals with CC 32, 422, $\overline{42} m$, and 622. In Fig. 2, these directions are shown by dashed lines. Breather zones (BZ) correspond to the hatched regions around these directions, where any one of the MFB will be most effective. There are forbidden zones (FZ), located between and outside of the BZ, where the MFB are not, or are only very weakly, effective.

In the general case, the number of characteristic directions depends on the symmetry of the crystals. Analyzing the expression (12), we can separate all quadratic uniaxial crystals into the three groups. The first group (F1) contains the crystals with the $\mathrm{CC} 4, \overline{4}, 4 \mathrm{~mm}, 6,6 \mathrm{~mm}$ of the tetragonal, and the hexagonal crystals, for which the component $\chi_{y y y}=0$. The 

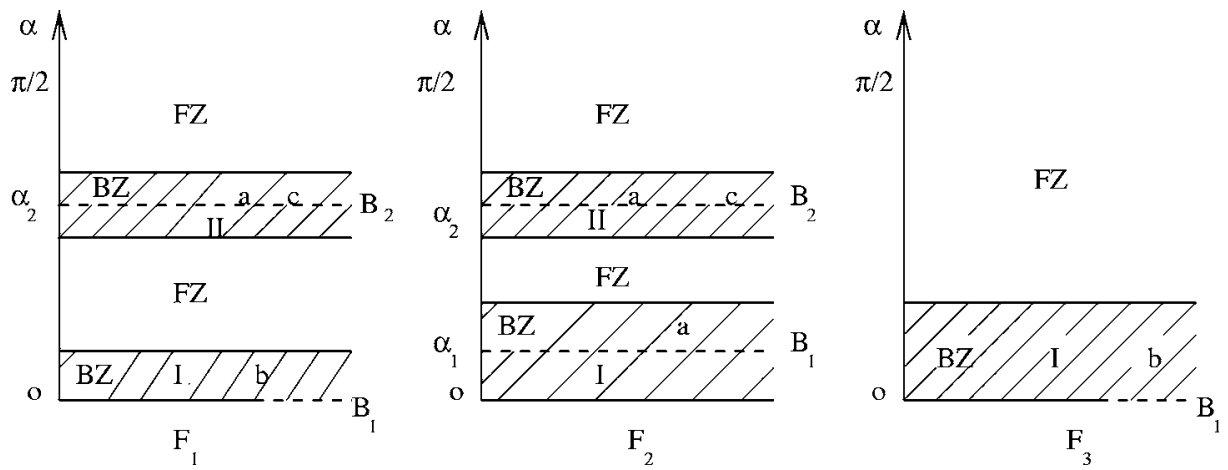

FIG. 3. There are three different SBZ realizations for the F1, F2, and F3 of crystals with quadratic nonlinearity. For crystals with cubic nonlinearity the SBZ coincides with the SBZ of F2 of the quadratic nonlinearity crystals. The optical breathers of the small area are formed in the zones I and II (BZ) by means of three different (a), (b), and (c) mechanisms as shown in the figures. These zones are hatched. The width of the zones depends on the nonlinearity parameters.

second group (F2) contains the crystals with the CC $3,3 m, \overline{6}$, and $\overline{6} m 2$ of the trigonal and hexagonal crystal systems, for which the component $\chi_{y y y} \neq 0$. The third group (F3) contains the crystals with the CC $32,422, \overline{42} \mathrm{~m}$, and 622 of the trigonal, tetragonal, and hexagonal crystal systems.

The situation considered in Fig. 2 corresponds to the F1 group [taking into account that for this group of crystals $\left.\chi_{l, l^{\prime}}^{(2)}(\alpha \rightarrow 0)=0\right]$ while the characteristic direction $B_{1}$ points along the $z$ axis. In order to investigate the dependence of MFB on the direction of the wave propagation, it will be more convenient for us to consider the first sketch (labeled F1) of the SBZ in Fig. 3.

Unlike the group F1, the group F2 realizes another SBZ (see Fig. 3, second sketch). In particular, for the group F2, the quantity $\chi_{l, l^{\prime}}^{(2)}(\alpha \rightarrow 0) \neq 0$ and consequently the characteristic direction $B_{1}$ does not point along the $z$ axis any more. For F2 crystals in the zone I, the direction $B_{1}$, at the angle $\alpha_{1}$, appears which is defined through the equation $M_{l}=R_{l, 0}$ (but for a different value $\chi_{l, l^{\prime}}^{(2)} \sim \chi_{y y y}$ than in the zone II). In zone II, resonant optical breathers will be formed by means of the mechanism (a), but resonant breather parameters in zones I and II will be different. When one has the condition $m_{l} \gg R_{l, 0}$ satisfied in zone II, then nonresonant optical breathers can also be formed by means of the mechanism (c).

Note that for the different groups of crystals (F1, F2, and F3), the directions $B_{1}$ and $B_{2}$ (the angles $\alpha_{1}$ and $\alpha_{2}$ ) will differ. For the F3 group, the situation is quite different as compared to F1 and F2 (see Fig. 3). In this case, all components of the quantity $\chi_{l, l^{\prime}}^{(2)}$ equal zero, and consequently $M_{l}=0$ everywhere. Then we have only one special direction $B_{1}$ which points along the $z$ axis. The quantity $R_{l, 0}(0)$ has its maximum value in this direction, meaning that in this single BZ the (b) MFB (i.e., SIT) will be realized. Of course, in the above considerations, we have always ignored any influence of the weaker cubic nonlinearity in comparison to the quadratic nonlinearity in our study of the SBZ in these crystals since when the quadratic nonlinearity is nonzero, it will normally dominate.

From Eq. (13) it follows that all uniaxial crystals with cubic nonlinearity (Kerr media) have the same characteristic directions and SBZ as those of the crystals of F2 with quadratic nonlinearity and hence Fig. 2, F2 applies for crystals with cubic nonlinearity as well. Note that mechanisms (b) and (c) do not act independently but also influence each other. They can support each other not only under the condition of (a) MFB being present, but also when $m_{l}+R_{l, 0}=0$. For example, in an amplifier media, for early times, the impurity atoms will be in excited states where $\tau_{0}=-1$. In this case, the mechanism (a) is not realized and consequently the SBZ will be changed significantly: In particular, in F1 of Fig. 3, the BZ II will become an FZ, and also in F2 of Fig. 3, the BZ's I and II will also be transformed into FZ's.

Consequently in the anisotropic uniaxial media three mechanisms [(a), (b), and (c)] of the formation of the optical breathers can be realized for different directions of the extraordinary wave propagation depending on the symmetry of the medium. The uniaxial crystals with quadratic nonlinearity can be divided into three different groups with each of them having its own SBZ. The SBZ within one of these groups does not depend on the crystallographic system and is determined by means of the CC. Unlike quadratic media for uniaxial crystals with cubic nonlinearity, the SBZ does not depend either on the crystal systems or on the CC, and one single SBZ is realized, which coincides with the SBZ of the F2 of the crystals with quadratic nonlinearity and with the CC $3,3 m, \overline{6}, \overline{6} m 2$ of the trigonal and hexagonal crystal systems. Hence the angular dependence of the mechanisms of the formation of breathers on the direction of the pulse propagation is qualitatively different for media with quadratic and cubic susceptibilities.

The SBZ for the case when the principal optical axis $O$ of the uniaxial crystal and the vector of electrical dipole moment of the impurity atoms $\vec{d}_{0}$ are parallel to the $z$ axis investigated in Refs. [5-7] in detail. Comparing results of this section with the results of Ref. [6], it is shown that SBZ essentially depends from the mutual orientation of the principal optical axis $O$ of the uniaxial crystal and the vector of electrical dipole moment of the impurity atoms. In order to make such comparison, in the next section, we will present the results of Ref. [6] in a convenient form for such a comparison. 

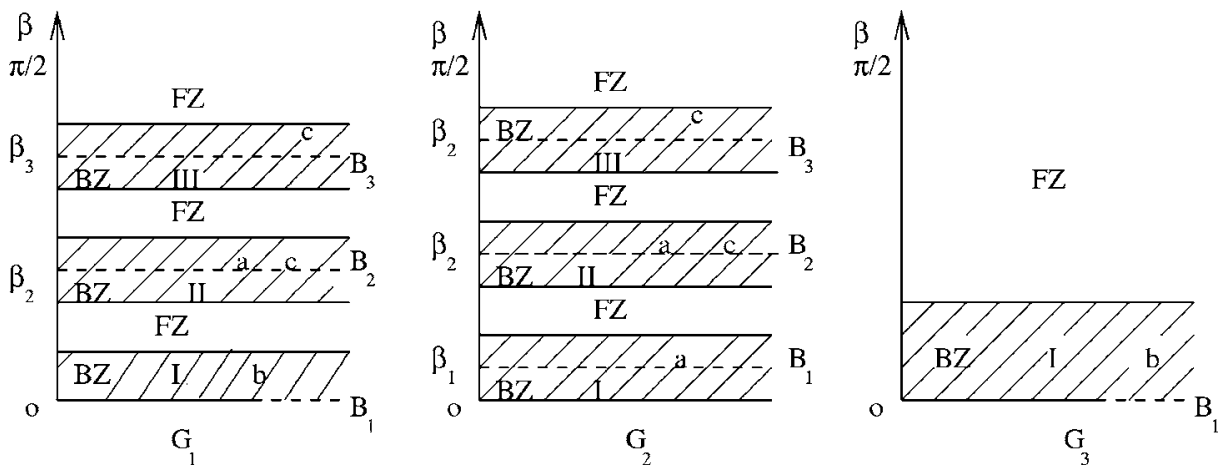

FIG. 4. There are three different SBZ realizations for the G1, G2, and G3 of crystals with quadratic nonlinearity for different value of the angle $\beta=\pi / 2-\alpha$. For crystals with cubic nonlinearity the SBZ coincides with the SBZ of G2 of the quadratic nonlinearity crystals. The optical breathers of the small area are formed in the zones I, II, and III (BZ) by means of three different (a), (b), and (c) mechanisms as shown in the figures. These zones are hatched. The width of the zones depends on the nonlinearity parameters.

\section{SBZ WHEN $\vec{d}_{0}$ POINTS ALONG AXIS $z$}

The effective susceptibilities of the second and third order in uniaxial media under the condition when the principal optical axis $O$ of the uniaxial crystal and the vector of electrical dipole moment of the impurity atoms $\vec{d}_{0}$ are parallel to the $z$ axis, have the following form:

$$
\begin{aligned}
\chi_{2, l, l^{\prime}}^{(\|)}(\alpha)= & 4 \pi l^{2} \omega^{2}\left\{\varphi _ { l } ( \alpha ) \left[\chi_{z z z}-\cot \alpha \Lambda_{l}\left(\chi_{z y z}+\chi_{z z y}\right)\right.\right. \\
& \left.+\cot ^{2} \alpha \Lambda_{l}^{2} \chi_{z y y}\right]-\mu_{l}\left[\cos \alpha \sin \alpha \chi_{y z z}\right. \\
& \left.\left.-\cos ^{2} \alpha \Lambda_{l}\left(\chi_{y y z}+\chi_{y z y}\right)+\cos ^{2} \alpha \cot \alpha \Lambda_{l}^{2} \chi_{y y y}\right]\right\},
\end{aligned}
$$

$$
\begin{aligned}
\rho_{3, l, l^{\prime}, l^{\prime \prime}}^{(I I)}(\alpha) & \\
= & 4 \pi l^{2} \omega^{2}\left\{\varphi _ { l } ( \alpha ) \left[\hat{\rho}_{z z z z}-\cot \alpha \Lambda_{l}\left(\hat{\rho}_{z y z z}+\hat{\rho}_{z z z y}+\hat{\rho}_{z z y z}\right)\right.\right. \\
& \left.+\cot ^{2} \alpha \Lambda_{l}^{2}\left(\hat{\rho}_{z y y z}+\hat{\rho}_{z y z y}+\hat{\rho}_{z z y y}\right)-\cot ^{3} \alpha \Lambda_{l}^{3} \hat{\rho}_{z y y y}\right] \\
& -\mu_{l}\left[\sin \alpha \cos \alpha \hat{\rho}_{y z z z}-\cos ^{2} \alpha \Lambda_{l}\left(\hat{\rho}_{y y z z}+\hat{\rho}_{y z z y}+\hat{\rho}_{y z y z}\right)\right. \\
& +\cos ^{2} \alpha \cot \alpha \Lambda_{l}^{2}\left(\hat{\rho}_{y y y z}+\hat{\rho}_{y y z y}+\hat{\rho}_{y z y y}\right) \\
& \left.\left.-\cos ^{2} \alpha \cot ^{2} \alpha \Lambda_{l}^{3} \hat{\rho}_{y y y y}\right]\right\}
\end{aligned}
$$

where

$$
\Lambda_{l}=\frac{\varepsilon_{\|}(l k, l \omega)}{\varepsilon_{\perp}(l k, l \omega)}, \quad \mu_{l}=\frac{c^{2} k^{2}}{\omega^{2} \varepsilon_{\perp}(l k, l \omega)}, \quad \varphi_{l}(\alpha)=1-\mu_{l} \cos ^{2} \alpha .
$$

The quantity $\mu_{l}$ from the dispersion relation for extraordinary waves Eq. (23) is thereby determined.

To take into account that in hexagonal, tetragonal, and trigonal crystals $\chi_{y z z}=\chi_{z y z}=\chi_{z z y}=0$ the expression (28) transformed to the form

$$
\begin{aligned}
\chi_{2, l, l^{\prime}}^{(I)}(\alpha)= & 4 \pi l^{2} \omega^{2}\left\{\varphi_{l}(\alpha)\left[\chi_{z z z}+\cot ^{2} \alpha \Lambda_{l}^{2} \chi_{z y y}\right]\right. \\
& \left.+\mu_{l}\left[\cos ^{2} \alpha \Lambda_{l}\left(\chi_{y y z}+\chi_{y z y}\right)-\cos ^{2} \alpha \cot \alpha \Lambda_{l}^{2} \chi_{y y y}\right]\right\} .
\end{aligned}
$$

To take into account that in trigonal, tetragonal, and hexagonal crystals with susceptibilities of the third order the terms $\hat{\rho}_{z y z z}=\hat{\rho}_{z z z y}=\hat{\rho}_{z z y z}=\hat{\rho}_{y z z z}=0$, the effective susceptibilities of the third order Eq. (29) transforms to the form

$$
\begin{aligned}
\rho_{3, l, l^{\prime}, l^{\prime \prime}}^{(\|)}(\alpha) & \\
= & 4 \pi l^{2} \omega^{2}\left\{\varphi _ { l } ( \alpha ) \left[\hat{\rho}_{z z z z}+\cot ^{2} \alpha \Lambda_{l}^{2}\left(\hat{\rho}_{z y y z}+\hat{\rho}_{z y z y}+\hat{\rho}_{z z y y}\right)\right.\right. \\
& \left.-\cot ^{3} \alpha \Lambda_{l}^{3} \hat{\rho}_{z y y y}\right]+\mu_{l}\left[\cos ^{2} \alpha \Lambda_{l}\left(\hat{\rho}_{y y z z}+\hat{\rho}_{y z z y}+\hat{\rho}_{y z y z}\right)\right. \\
& -\cos ^{2} \alpha \cot \alpha \Lambda_{l}^{2}\left(\hat{\rho}_{y y y z}+\hat{\rho}_{y y z y}+\hat{\rho}_{y z y y}\right) \\
& \left.\left.+\cos ^{2} \alpha \cot ^{2} \alpha \Lambda_{l}^{3} \hat{\rho}_{y y y y}\right]\right\} .
\end{aligned}
$$

This expression is valid for the trigonal, tetragonal, and hexagonal crystals.

In special case, only in the tetragonal and hexagonal crystals, in which the terms $\hat{\rho}_{y y y z}=\hat{\rho}_{y y z y}=\hat{\rho}_{y z y y}=\hat{\rho}_{z y y y}=0$, Eq. (31) becomes

$$
\begin{aligned}
\rho_{3, l, l^{\prime}, l^{\prime \prime}}^{(\|)}(\alpha) & \\
= & 4 \pi l^{2} \omega^{2}\left\{\varphi_{l}(\alpha)\left[\hat{\rho}_{z z z z}+\cot ^{2} \alpha \Lambda_{l}^{2}\left(\hat{\rho}_{z y y z}+\hat{\rho}_{z y z y}+\hat{\rho}_{z z y y}\right)\right]\right. \\
& +\mu_{l}\left[\cos ^{2} \alpha \Lambda_{l}\left(\hat{\rho}_{y y z z}+\hat{\rho}_{y z z y}+\hat{\rho}_{y z y z}\right)\right. \\
& \left.\left.+\cos ^{2} \alpha \cot ^{2} \alpha \Lambda_{l}^{3} \hat{\rho}_{y y y y}\right]\right\} .
\end{aligned}
$$

Using Eqs. (30)-(32) we can construct the SBZ under the condition when the principal optical $O$ axis of the uniaxial crystal and the vector of electrical dipole moment of the impurity atoms $\vec{d}_{0}$ are pointed along the $z$ axis. In this case, unlike in Ref. [6], we will construct the SBZ for three groups of quadratic uniaxial crystals, not for angle $\alpha$, but for the angle $\beta=\pi / 2-\alpha$. The first group (G1) contains the crystals with the CC $\overline{4}, \overline{6}$, and $\overline{6} m 2$ of the uniaxial tetragonal and hexagonal crystal systems; the second group (G2) contains the crystals with the CC $3,3 \mathrm{~m}, 4,4 \mathrm{~mm}, 6,6 \mathrm{~mm}$ of the trigonal, tetragonal, and hexagonal crystal systems; and the third group (G3) contains the crystals with the CC 32, 422, $\overline{42} \mathrm{~m}$, and 622 of the trigonal, tetragonal, and hexagonal crystal systems. Analogous to the previous section, we can construct SBZ for this case (see Fig. 4).

\section{CONCLUSION}

In an anisotropic uniaxial media, there are three mechanisms [(a), (b), and (c)] by which the formation of the optical 
breathers can be realized, for different directions of the extraordinary wave propagation, depending on the symmetry of the medium, the orientation of the principal optical axis $O$ of the uniaxial crystal, and the vector of electrical dipole moment of the impurity atoms $\vec{d}_{0}$. We find that there are two interesting cases, which are when the principal optical axis $O$ of the uniaxial crystal and the vector of electrical dipole moment of the impurity atoms $\vec{d}_{0}$, are perpendicular or parallel to each other. Corresponding results for these cases are given in Secs. IV and V. In both cases the uniaxial crystals with quadratic nonlinearity can be divided into three different groups, with each of them having its own SBZ. The SBZ within any one of these groups does not depend on the crystallographic system and is determined by the CC.

On the other hand, for uniaxial crystals with cubic nonlinearity, the SBZ does not depend on either the crystal systems or on the CC, and one single SBZ is realized, which coincides with the SBZ of F2 in Fig. 3 (with the CC $3,3 m, \overline{6}$, $\overline{6} m 2$ of the trigonal and hexagonal crystal systems, if $\hat{\rho}_{z z z z}>0$ ), when optical axis $O \perp \vec{d}_{0}$ and coincides with the SBZ of the G2 in Fig. 4 when the optical axis $O \| \vec{d}_{0}$. Hence the angular dependence of the mechanisms of the formation of breathers on the direction of the pulse propagation is qualitatively different for media with quadratic and cubic susceptibilities.

We can divide the results into three different groups in these two cases. The F1 $(4 \overline{4}, 4 \mathrm{~mm}, 6,6 \mathrm{~mm})$ and G1 $(\overline{4}, \overline{6}$, and $\overline{6} m 2)$, and F2 $(3,3 m, \overline{6}$, and $\overline{6} m 2)$ and $\mathrm{G} 2(3,3 m, 4$, $4 \mathrm{~mm}, 6,6 \mathrm{~mm}$ ) groups contain crystals with different $\mathrm{CC}$, but F3 and G3 group contains the same crystals with the CC 32, 422, $42 m$, and 622 .

Comparing Figs. 3 and 4, we see that groups F1 and F2 contain only two different BZ, but groups $\mathrm{G} 1$ and $\mathrm{G} 2$ contain three different BZ's. For the F1 and G1 groups, BZ I and II happen to coincide. But F1 does not contain BZ III when one only has nonresonant MFB (c). Analogously for the F2 and G2 groups, BZ's I and II coincide, but F2 does not contain the BZ III when one only has nonresonant MFB (c). The same situation is true also for all crystals with cubic order. The SBZ for F3 and G3 groups coincide.
Once one has these possible BZ's delineated, then the existence of breathers in any BZ will still crucially depend on the sign of $p q$. It is only when $p q>0$ that breathers can occur, which is fulfilled whenever $m_{l}$ is positive. But sometimes, some components of $\chi_{i j k}$ can be also negative (see, for example, Ref. [20]). In connection with this, it is an interesting situation if $\chi_{z z z}<0$. In this case, under the condition when $O \perp \vec{d}_{0}$, the corresponding quantity $\chi_{l, l^{\prime}}^{(2)}(\alpha \rightarrow / \pi 2)$ will be positive. However if $M_{l} w_{2 l, 2 l}>0$, for $\alpha$ close to $\pi / 2$ nonresonant MFB (c) can be realized, i.e., for the crystals F1 and F2 we can have BZ III with MFB (c). This statement is valid only for the crystals F1 and F2 with the CC 3, 3m, 4, 4mm, $6,6 \mathrm{~mm}$ of the trigonal, tetragonal, and hexagonal crystal systems for which $\chi_{z z z} \neq 0$, but not for crystals F1 and F2 with $\mathrm{CC} \overline{4}, \overline{6}$, and $\overline{6} m 2$ of the tetragonal and hexagonal crystal systems for which $\chi_{z z z}=0$, BZ III with MFB (c) always are absent (Fig. 3, F1 and F2).

This remark is valid also for the crystals with cubic susceptibilities in which the only SBZ is similar to F2 in Fig. 3 with the CC $3,3 m$ of the trigonal crystal system if $\hat{\rho}_{z z z z}<0$. In this case, under the condition when $O \perp \vec{d}_{0}$, the quantity $\rho_{l, l^{\prime}, l^{\prime \prime}}^{(3)}(\alpha \rightarrow \pi / 2)$ and $L_{l}$ will be positive. Then for $\alpha$ close to $\pi / 2$, nonresonant MFB (c) will dominate, i.e., for the crystals with cubic susceptibilities, there will appear a BZ with MFB (c).

Consequently, SBZs of the different groups of crystals are dependent not only on the direction of propagation of the extraordinary wave, but also on the symmetry of the medium, as well as on the mutual orientations of the principal optical axis $O$ of the uniaxial crystal and the vector of electrical dipole moment of the impurity atoms $\vec{d}_{0}$. For the cases where the principal optical axis of the uniaxial crystal and the vector of electrical dipole moment of the impurity atoms are either parallel or perpendicular, the SBZs are significantly different.

\section{ACKNOWLEDGMENTS}

G.T.A. thanks the MPIPKS for hospitality. This research has been supported in part by NSF Grant No. DMS-0129714.
[1] G. T. Adamashvili and A. A. Maradudin, Phys. Rev. E 55, 7712 (1997).

[2] S. L. McCall and E. L. Hahn, Phys. Rev. 183, 457 (1969).

[3] G. T. Adamashvili and S. V. Manakov, Solid State Commun. 48, 381 (1983).

[4] G. T. Adamashvili, Opt. Spektrosk. 176, 1400 (1992); 76, 3 (1994); 80, 251 (1996).

[5] G. T. Adamashvili, Phys. Lett. A 203, 473 (2003).

[6] G. T. Adamashvili, Phys. Rev. E 69, 026608 (2004).

[7] G. T. Adamashvili and D. J. Kaup, Phys. Rev. E 70, 066616 (2004).

[8] As a point of clarification, there have been two different definitions of "breathers" in the literature, which do cause confu- sion. The original definition of "breather" was a bound state of a kink-antikink in the Sine-Gordon equation. This is a twosoliton solution of the Sine-Gordon equation [9], and is manifestly stable against infinitestimal perturbations of the initial data. On the other hand, certain two-soliton solutions of other integrable equations, such as the NLS, the modified KdV equation, etc., have also been called "breather" solutions, provided that each soliton in the breather had exactly the same velocity, such that they would travel as a unit. Although these breather solutions would indeed travel as a unit and would oscillate, they were also manifestly unstable to almost any infinitesimal perturbation of the initial data, upon which they would slowly separate, since the binding energy was zero. Here, we are using 
the original definition of the breather, as being a stable, bound state of two kinklike solitons. In the low amplitude limit, the envelope of this breather is described by a one-soliton solution of the NLS.

[9] M. J. Ablowitz, D. J. Kaup, A. C. Newell, and H. Segur, Phys. Rev. Lett. 30, 1262 (1973).

[10] A. C. Newell, Solitons in Mathematics and Physics (Society for Industrial and Applied Mathematics, Philadelphia, PA, 1985).

[11] Min Chen, D. J. Kaup, and B. Malomed, Phys. Rev. E 69, 056605 (2004).

[12] A. A. Kaminskii, Lasernie Kristali (Nauka, Moscow, 1975).

[13] E. G. Sauter, Nonlinear Optics (Wiley, New York, 1996).

[14] A. C. Newell and J. V. Moloney, Nonlinear Optics (AddisonWesley, New York, 1992).

[15] M. B. Vinogradova, O. V. Rudenko, and A. P. Suhorukov, Theoria Voln (Nauka, Moscow, 1990).

[16] V. M. Agranovich and V. L. Ginzburg, Kristallooptika s Uchotom Prostranstvennoi Dispersii i Teoria Eksitonov (Nauka, Moscow, 1979).

[17] A. S. Davidov, Teoria Tverdogo Tela (Nauka, Moscow, 1979).

[18] V. M. Agranovich, G. T. Adamashvili, and V. I. Rupasov, Zh.
Eksp. Teor. Fiz. 80, 1741 (1981).

[19] A. Yariv and P. Yeh, Optical Waves in Crystals (Wiley, New York, 1984).

[20] Nelineinie Svoistva Tverdich Tel, edited by V. M. Fain (Mir, Moscow, 1972).

[21] L. Allen and J. N. Eberly, Optical Resonance and Two Level Atoms (Wiley-Interscience, New York, 1975).

[22] A. I. Maimistov, A. M. Bahsarov, S. O. Elyutin, and Yu. M. Sklyarov, Phys. Rep. 191, 1 (1990).

[23] T. Taniuti and N. Iajima, J. Math. Phys. 14, 1389 (1973).

[24] R. K. Dodd, J. C. Eilbeck, J. D. Gibbon, and H. C. Morris, Solitons and Nonlinear Wave Equation (Academic Press, New York, 1982).

[25] V. E. Zaharov, S. V. Manakov, S. P. Novikov, and L. P. Pitaevski, Teoria Solitonov, Metod Obratnoi Zadachi (Nauka, Moscow, 1980).

[26] M. Ablowitz and H. Segur, Solitons and the Inverse Scatering Transform (SIAM, Philadelphia, 1981).

[27] D. J. Kaup, Phys. Rev. A 16, 704 (1977).

[28] D. J. Kaup and A. C. Newell, Proc. R. Soc. London, Ser. A 361, 413 (1978). 